\title{
Role of the mental foramens in dolphin hearing
}

\author{
Vyacheslav Ryabov
}

Karadag Natural Reserve NAS of Ukraine, Feodosia, Crimea, Ukraine; ryaboff@ukr.net

Received 4 March 2010; revised 16 April 2010; accepted 13 May 2010.

\section{ABSTRACT}

The role of mental foramens in dolphin hearing was studied in the present work. To this effect the mental foramens' morphology features which are essential from acoustical viewpoint have been studied. The patterns of relationship between the location of mental foramens and their sizes are found. The affinity of the mental foramens' morphology and acoustics that the nature had created testify acoustical expediency of the mental foramens' architecture. This natural inference in the main is confirmed in this work by the experimental data. The mean values of detection thresholds of short broadband stimuli with spectral maximum on frequencies 8 , 16,30 and $100 \mathrm{kHz}$ at acoustical shielding the mental foramens increased on 30, 34, 40 and $50 \mathrm{~dB}$, respectively. Results obtained testify that the mental foramens are the unique sound-conducting pathway into the fat body of the mandibular canal for the sounds of all frequencies used in the experiment, approximately $6-150 \mathrm{kHz}$ (in view of stimuli broadbandness). The left and right row of the mental foramens together with respective mandibular canal plays the role of pinna and external auditory meatus if to use conventional terminology of a land mammal ear. But it is already qualitatively the new external ear implemented by the nature as the receive array and acoustical horn. The new external ear has apparently appeared in result of the dolphins' ancestors' adaptation to new environment conditions, as evolutionary adaptation of the ear to the water and as functional adaptation of the ear in order that to fulfill the new more sophisticated functions in the structure of sonar. The findings give good reason to suppose existence of the same external ear in Odontoceti.

Keywords: Dolphin; Hearing; Architecture of Mental Foramens; Lower Jaw; Unique Pathway; Sound Conduction; The Traveling Wave Antenna; External Ear

\section{INTRODUCTION}

The mechanisms of Odontoceti hearing attract researchers for many years, therefore, a lot of works have been performed in this study area and different hypotheses about sound-conduction mechanisms to a cochlea were suggested in the works. Some researchers assume that the sound passes to the cochlea through the external auditory meatus and the middle ear [1-3]. However, there is an opinion that the auditory meatus cannot participate in sound transmission to the middle ear in general [4-6], or transmits sounds with frequencies under $30 \mathrm{kHz}[7,8]$. On the grounds of hypothesis related to the sound conduction through the external auditory meatus, authors discuss the possibility of functionally specific subsystems of passive (1-10 kHz) and active (about $100 \mathrm{kHz}$ ) hearing [9].

Norris [10] supposed that the sound can be conducted into the fat body of mandibular canal through the mental foramens. Though, little later he suggests different hypothesis [11] which is being generally accepted so far, to the effect that the sound passes into the mandibular fat body directly through the postero-lateral wall of mandible in the area which he called the "acoustic window".

Two "acoustic windows" were determined in electrophysiological experiments: one is for low frequencies $(16-22.5 \mathrm{kHz})$, which is located in the area of external auditory meatus, and the other one - for high frequencies $(32-128 \mathrm{kHz})$ which is located at a distance of $9.3-13.1 \mathrm{~cm}$ from melon tip (i.e. proximal part of a lower jaw) [12].

It has been also revealed that the fat body conducts the sound to the lateral wall of the tympanic bone, where its thickness is minimum, and the wall play a part of the tympanic membrane, transmitting acoustical vibration to the malleus of the middle ear $[6,7,10,11,13-16]$. Acoustic stimulation of a lower jaw excites considerable evoked potentials in the dolphin's central auditory system [7]. However, the areas of maximum sensitivity of the mandible surface to sounds of the contact point emitter (size smaller than wavelength) in each work are different, and the test results do not explain the sound-conduction me- 
chanisms $[6,7,17,18]$.

Acoustic shielding of the lower jaw significantly hindered the dolphin's ability to discriminate between targets in the echolocation task [19]. Results of these experiments support hypothesis that the lower jaw has a role in the reception of high frequency signals and their transmission to the middle and inner ear. However, the acoustical shield covers the lower jaw from the rostrum tip up to the bases of pectoral fins, i.e. it covers both mental foramens, and "acoustic windows", and ventral area of the head, therefore the pathways and mechanisms of sound-conduction so far remain unclear. Along with this, the possibility of simultaneous participation of the auditory meatus and "acoustic windows" for sound conduction to the cochlea at forming spatial auditory image by a dolphin were also discussed [20].

Thus, results presented in the review are inconsistent and the main questions about the pathways and mechanisms of sound-conduction still remain without answers. At the same time the results [21-23] give good reason to consider the mental foramens as the unique sound-conduction pathway into the fat body of dolphin's mandibular canal. The morphology analysis of the dolphin's lower jaw and subsequent modeling confirm this assumption and afford ground to assume that the left and right row of mental foramens from acoustical viewpoint represents the traveling wave antenna which is located in the throat of acoustical catenoidal horn (the left and right mandibular canal plays a role of horn). The model with two traveling wave antennas explains mechanisms of the sound-conduction and directivity of the peripheral part of dolphin's auditory system [21-25]. In agreement with this, anatomical structures of each half of the lower jaw (mental foramens, mandibular canal and fat body) are considered as the components of this part.

We study the role of mental foramens in the dolphin hearing through experiments. The specific tasks of this work are study of the morphology features of mental foramens that are essential from acoustical viewpoint and experimental measurement of the detection thresholds dependence of wide-band acoustical impulses with spectral maximum on different frequencies at mental foramens' acoustical shielding.

\section{METHODS}

\subsection{Subject and Experimental Conditions}

The mandible bones of adult bottlenose dolphin (Tursiops truncatus $p$.) were used for studying the morphology of mental foramens. In order to perform the measurements the mandible was sawn in the area of mental foramens. Further in the text if it was necessary, mental foramens was indicated with the number, as $M F n$, where $n$-is the number of foramen, counting from the rostrum tip; $n=1,2,3,4$. The cross section dimensions of mental foramens were measured in the plane of maximum width of each foramen, and then in the mutually perpendicular plane.

The experimental studies were carried out with adult Black Sea dolphin (Tursiops truncatus p.) which earlier never participated in acoustic experiments, on the base of Karadag Natural Reserve of NAS of Ukraine in the indoor pool of $27.5 \times 9.5 \times 4.5 \mathrm{~m}$.

\subsection{Experimental Procedure}

The experiments were carried out with using behavioral response techniques (operant conditioning with food reinforcement). A go/no-go response paradigm [26] was used for reporting stimuli condition. The experimental facilities were laid out as follows (Figure 1). The dolphin was trained to approach the testing platform (1) upon a trainer's signal, where the trainer was putting on (or was not putting on) the acoustic shield (Figure 2) on its rostrum (mental foramens' area). After that the dolphin upon the trainer's signal approached the start ball, and stood touching it by the rostrum tip. Then the researcher switched on the stimulus (or not switched on the stimulus). The stimuli were presented over a period of 4 seconds with repetition frequency $3 /$ second. The dolphin reported that detects the produced stimulus by leaving the start position within $4 \mathrm{~s}$ of the trial beginning and touching the signal ball with his rostrum, or if the stimulus was not produced by remaining on the start position within $4 \mathrm{~s}$ till a trainer's signal.

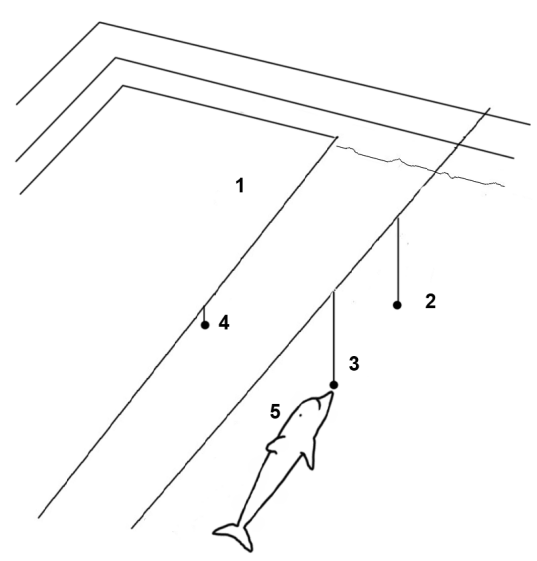

Figure 1. The experiment configuration. 1-the testing platform, 2-the stimulus transmitter, 3-the start ball, 4-the signal ball, 5 -the dolphin in start position. The dolphin, start ball and stimulus transmitter are located $1 \mathrm{~m}$ below the water surface. Signal ball is located near the water surface. The distance between transmitter and pool wall is $3 \mathrm{~m}$, the distance between transmitter and start ball is $2 \mathrm{~m}$. 
In these cases the dolphin receives a fish reward for the correct response. The acoustic shield was removed by the trainer every time if it was needed for fish reward. The errors (either dolphin was not approached to the signal ball when signal was produced, or was approached to the signal ball when signal was not produced - false alarm) were not rewarded.

The producing or not producing of stimuli in each trial was determined in random order (but not more than three similar stimuli in sequence). From trial to trial, the stimuli level varied by a one-up-one-down staircase procedure. The session began from a stimuli level well above the anticipated threshold the warming-up part of the session. From trial to trial, the stimuli level varied according to the animal's response in the preceding trial. If the animal detected the stimuli, the level in the next trial decreased one step down. If the animal missed the stimuli, the stimuli level in the next trial increased one step up. The steps were $6 \mathrm{~dB}$ in the warming-up part of the session, until the first miss. After that, the steps were $3 \mathrm{~dB}$ the measurement part of the session. Responses in stimulus-absent trials did not influence the level in stimulus-present trials. The last one or two trials were again well above the threshold to make sure of ending the session with rewards the cooling-down part of the session. About twenty trials were performed at each step of intensity variation. The threshold was calculated as the mean of all reversal points' maxima and minima of the staircase succession.

\subsection{Instrumentation}

The short broad-band acoustical impulses were used to decrease the effect on experiments results of the direct and reflected signals interference in the pool. Spherical acoustic transducers of 50 or $20 \mathrm{~mm}$ diameter were excited by rectangular electrical impulses with the duration of 17 or $5 \mu \mathrm{s}$ in order to obtain stimuli (the simulated dolphin-like echo-location clicks) with the energy spectrum maximum on frequencies 30 or $100 \mathrm{kHz}$, respectively. In order to obtain acoustical impulses with the energy maximum on frequencies 8 or $16 \mathrm{kHz}$, the transducer of $50 \mathrm{~mm}$ diameter was excited by rectangular electrical impulse through octave-band filter with center frequency of 8 or $16 \mathrm{kHz}$, respectively. The duration of each stimulus did not exceed 3 periods of the frequency of its spectrum maximum. In this case the reflections from pool walls and surface of water did not overlap the stimulus, as they were coming with sufficient time delay and were significantly weaker in comparison with the direct signal. Thus, at the stimuli levels near the hearing thresholds, the reflections levels were lower than the detection thresholds of the stimuli. This allows performing the measurements without special sound absorbers.
The acoustically opaque shield of the mental foramens (Figure 2) was made as per the shape of dolphin's rostrum so that the shield was put on tightly on the rostrum; the length of the shield was about $15 \mathrm{~cm}$. The shield is produced of the foamed (with closed-cell) neoprene sheet of $5 \mathrm{~mm}$ thickness. This waterproof and oil-resistant material has enough durability. Therefore, the efficiency of sound-shielding that gas bubbles of the material provide remains the same for a long time. The acoustical shielding efficiency of this material was tested before the experimentation with using simulated dolphin-like echolocation clicks with spectral maximum on frequencies either 10 or 55 or $170 \mathrm{kHz}$. Shielding the hydrophone with this material decreased the peak sound pressure level of these dolphin-like clicks up to 28,32 and $36 \mathrm{~dB}$ respectively, for normal incidence of sound.

It is necessary to note, that in frequency range of stimuli used in this experiment the sound wave-length vary from 1.5 to $20 \mathrm{~cm}$, i.e. sizes of the acoustic shield on low frequencies are relatively small in comparison with the wave-length. Considering it, the shield covers the upper and lower jaw for providing the best sound shadow in the region of mental foramens (Figures 2 and 3). If to make the shield form as external surface of the mandible (i.e. shield only lower jaw), then necessary shielding efficiency of the mental foramens, apparently, cannot be received owing to sound diffraction even on the frequency $100 \mathrm{kHz}$ (the sound wave-length $1.5 \mathrm{~cm}$ ), because distance from edge of the shield up to the mental foramens in this case will be comparable with the wave length (Figure 3), let alone low frequencies.

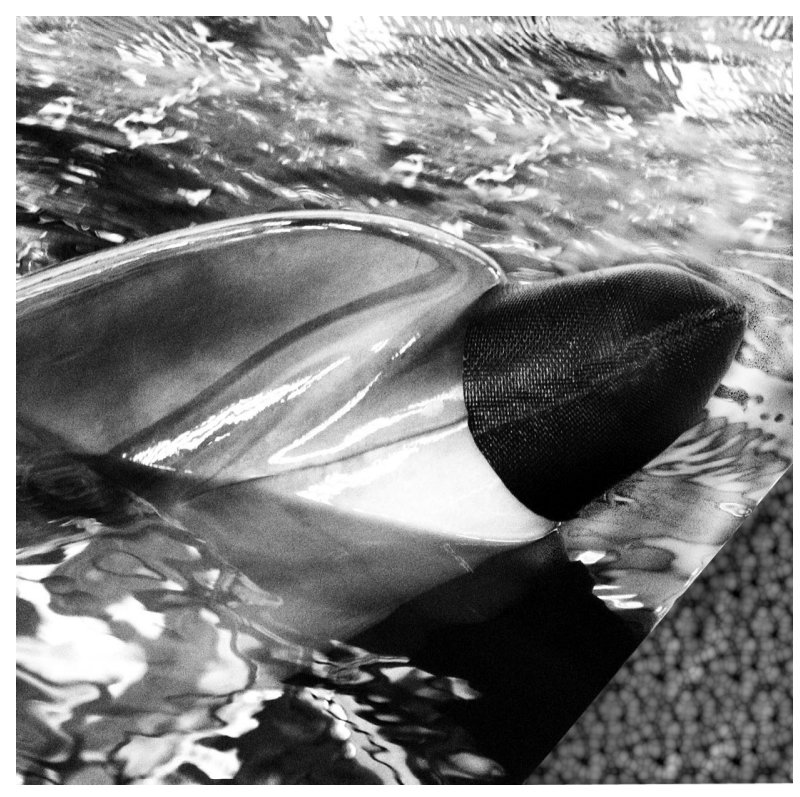

Figure 2. The dolphin is near testing platform with the acoustically opaque shield on his rostrum. 


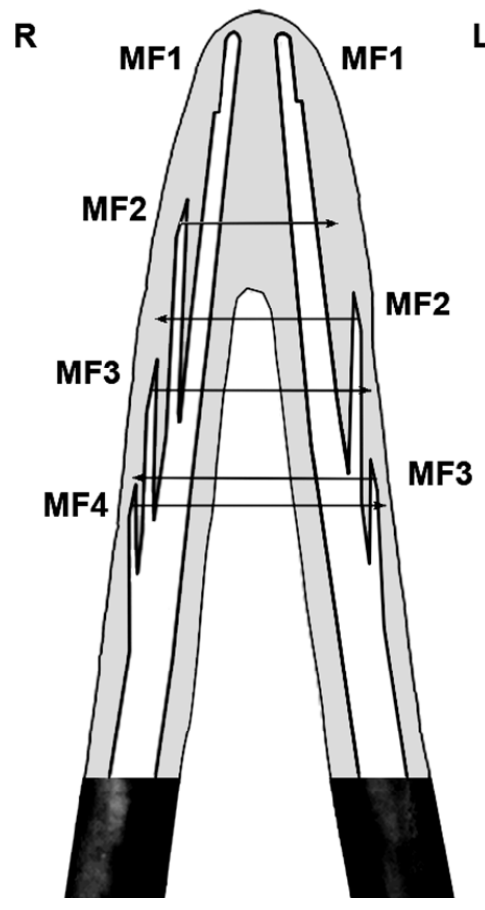

(a)

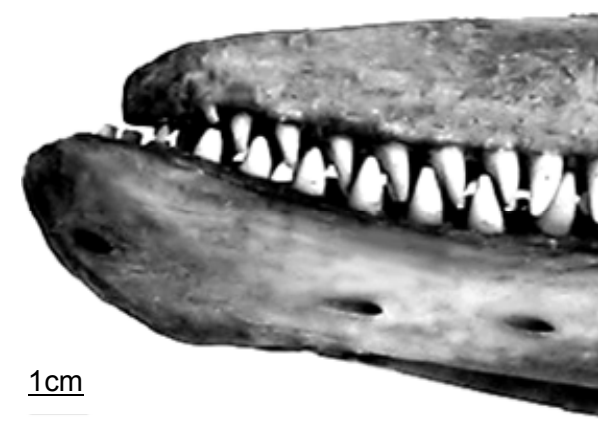

(b)

Figure 3. (a) The nasal part (symphysis) of the lower jaw shown in ventral view. In the cross section are presented the layout of MFs of the left (L) and right (R) half of the lower jaw and the region of lower jaw covered by the acoustical shield. The arrows show the projection of external orifices of MFs on the adjacent halves of the lower jaw; (b) The characteristic shape of the oblique endings of mental foramens shown on the left lateral side of the dolphin's rostrum.

\section{RESULTS}

\subsection{Acoustical Features of the Dolphin's Mental Foramens}

The lower jaw's morphology of Odontoceti is similar [11, 27]. The mandible is presented by two rectilinear hollow bones, connected together (in shape of the V or Y letter) in the nasal part along midline of the mandibular symphysis. The cavity of mandible is filled up with the fat body and the corresponding neurovascular bundle. In the area of symphysis the mandibular canal (MC) is pierced by the mental foramens in the places where the branches of mental nerve (n. mentalis) and blood-vessels go out of the mandibular canal on an external surface of mandible.

The cross section size of the neurovascular bundle is much smaller than the sounds wavelengths propagating along the mental foramens and the mandibular canal. The acoustic impedance of tissues filling lower jaw's canals is close to the impedance of sea water [28]. Hence these tissues do not introduce the acoustic heterogeneities and they are transparent for sound. The walls of mental foramens and mandibular canal are acoustically elastic. Using these preconditions, the sound conduction via mental foramens and mandibular canal has been analyzed on the basis of lower jaw's canals geometry [21, $22,23,25]$.

Let's consider in details the most essential features of structure, shape and sizes of the mental foramens of the dolphin (Tursiops truncatus p.) from acoustical viewpoint. The most vivid feature is the amount of the mental foramens. On the left half of the lower jaw there are three of them (Figure 3); on the right half - four, what are characteristically not only for the bottlenose dolphins, but for the others species of Odontoceti $[11,27]$.

The mental foramens have frontal direction and oblique ends. The oblique endings of first foramens are somewhat shorter as compared to the others. The distances between the mental foramens are decreasing with the distance from rostrum tip (Table 1). It's quite curious

Table 1. The mental foramens' basic dimensions of Black Sea dolphin (Tursiops truncatus p.). The first and second numbers in column "MF cross section area" are the values from external and internal end of the foramen, respectively. The lengths of MFs are indicated without taking of the oblique ends into account.

\begin{tabular}{|c|c|c|c|c|c|}
\hline $\begin{array}{c}\text { MF } \\
\text { number }\end{array}$ & $\begin{array}{c}\text { MF } \\
\text { length } \\
(\mathrm{mm})\end{array}$ & \multicolumn{2}{|c|}{$\begin{array}{c}\text { MF cross } \\
\text { section area } \\
\left(\mathrm{mm}^{2}\right)\end{array}$} & $\begin{array}{c}\text { Distance to } \\
\text { the next } \\
\mathrm{MF}(\mathrm{mm})\end{array}$ & $\begin{array}{l}\text { Length of } \\
\text { oblique end } \\
(\mathrm{mm})\end{array}$ \\
\hline \multicolumn{6}{|l|}{1} \\
\hline left & 10 & 8.04; & 8.5 & 50.2 & 7 \\
\hline right & 11 & 7.28; & 8 & 36.1 & 8 \\
\hline \multicolumn{6}{|l|}{2} \\
\hline left & 27.5 & 5 & 7.7 & 31.2 & 11 \\
\hline right & 35 & 4.29; & 6.04 & 31.5 & 13.5 \\
\hline \multicolumn{6}{|l|}{3} \\
\hline left & 12 & 5 & 5.7 & & 11 \\
\hline right & 20 & 4.12; & 4.94 & 19.15 & 11.5 \\
\hline \multicolumn{6}{|l|}{4} \\
\hline right & 10.5 & 1.53; & 1.53 & & 10 \\
\hline
\end{tabular}


that between the foramens' location and their sizes there are certain patterns of relationship. The mental foramens of the right half of lower jaw is longer than the foramens with the same number of the left half, i.e. if the length of mental foramens is denoted as $L_{m n}$, where $m$ is the left $(\boldsymbol{\ell})$ or right $(r)$ half of the lower jaw: $m=\ell$ or $r ; n$ is the index of the mental foramens: $n=1,2,3$ or 4 ; then, obviously, $L_{r 1}>L_{\ell 1}, L_{r 2}>L_{\ell 2}$ and $L_{r 3}>L_{\ell 3}$. Besides, if the foramen is further from the rostrum tip, the length of the foramen (except of MF1) is decreasing, i.e. $L_{r 2}>L_{\ell 2}$ $>L_{r 3}>L_{\ell 3}>L_{r 4}$. The length of MF1 in each half of the mandible is essentially shorter than the distance between external orifices of MF1 and MF2. At the same time the length of MF2 and MF3 in the right half of the mandible is few mm longer than the respective distances between external orifices of MF2-MF3 and MF3-MF4 (Table 1). Whereas, the length of MF2 in the left half is a few $\mathrm{mm}$ shorter than the distance between the orifices of MF2-MF3 (Figure 3). In the area of mental foramens the cross-sectional dimensions of the mandibular canal and its cross-sectional area gradually increases (caudal), in respect to each mental foramen. Whereas the cross-sectional dimensions of the mental foramens are decreasing with the distance from rostrum tip, though not as harmoniously as their lengths. Due to this the ratio of cross-sections areas of $M F n / M C$ in the area of mental foramens is decreasing (caudal).

But the most obvious and interesting is that the mental foramens of the left and right halves of the lower jaw (except of MF1) are located asymmetrically relatively to longitudinal axis of the animal, though the both halves of the lower jaw are symmetric. Due to the non-equidistant and asymmetrical locations of the mental foramens on both halves of the mandible the projection of each mental foramen (beginning from MF2) on the adjacent half of the lower jaw is located between respective mental foramens (arrows in the Figure 3), i.e. the location of mental foramens and their lengths are mutually complementally. This vivid result of morphology study obviously shows to us the affinity of acoustics and morphology of mental foramens that we see in their left-right mutually complementary asymmetry.

\subsection{Influence of Acoustic Shielding of Mental Foramens on Dolphin Hearing}

In the present study, we examined the effect of acoustical shielding of the mental foramens on the detection thresholds by the dolphin of the short broad-band sound stimuli with different spectrum maximums of $8,16,30$ and $100 \mathrm{kHz}$. The results of measurements are presented in form showing up the relative impairment of the dolphin's hearing at acoustical shielding of the mental foramens in dependence on the frequency of the stimuli spectrum maximum (Figure 4). In all tested frequency range $(6-150 \mathrm{kHz}$, taking into account the broadbandness of stimuli), the shielding efficiency is high and increases with the frequency from 30 to $50 \mathrm{~dB}$.

The sound-wavelength in water in dependence on frequency is presented on the same figure for best understanding of the results. The fact that the shielding efficiency dependence on the stimuli frequency is a sort of mirror reflection of the sound-wavelength dependence on frequency (Figure 4) proves that the shielding efficiency is inversely proportional to the stimuli wavelength and it means that the shielding efficiency is determined by the stimuli wavelength.

The absolute values of the detection thresholds of stimuli measured in this work agree with the bottlenose dolphin audiogram [29], taking into account phenomenon of the hearing energy summation [30,31]. Therefore, the dolphin, which was used in our experiment, has got normal hearing.

\section{DISCUSSION}

The studying of morphology features of the mental foramens was rather the successful but has created the new question. With what purpose the nature has created such complex harmony of mutually-complementary asymmetric architecture of the mental foramens? For the answer to this question it is necessary to take into account the fact that the left and right row of the mental foramens we considered like the traveling wave antenna and the lower jaw as a peripheral part of a dolphin's hearing system. Therefore we consider the studied morphology features of the mental foramens from viewpoint of the linear arrays' theory and physical acoustics. In this case [21-25], the mental foramens being acoustically narrow waveguides within the range of dolphin's hearing frequencies conduct the sound into the fat body of mandibular canal without distortion and define its intensity. The length of mental foramens and their location defines the delays of sound conduction in the traveling wave antenna. In other words, the mental foramens set the amplitude and phase distribution of particles velocities of the left and right antenna arrays. The oblique ends of the mental foramens, being the waveguides endings, adjust the active acoustical lengths of the mental foramens depending on frequency. The mental foramens are functioning as elementary receivers of arrays and the structure of their location defines the beam pattern of each antenna array. Therefore, mutually-complementary asymmetric architecture of the mental foramens is apparently necessary in order that to form the features of beam patterns of the left and right arrays. The preliminary calculations point out that beam patterns of the left 


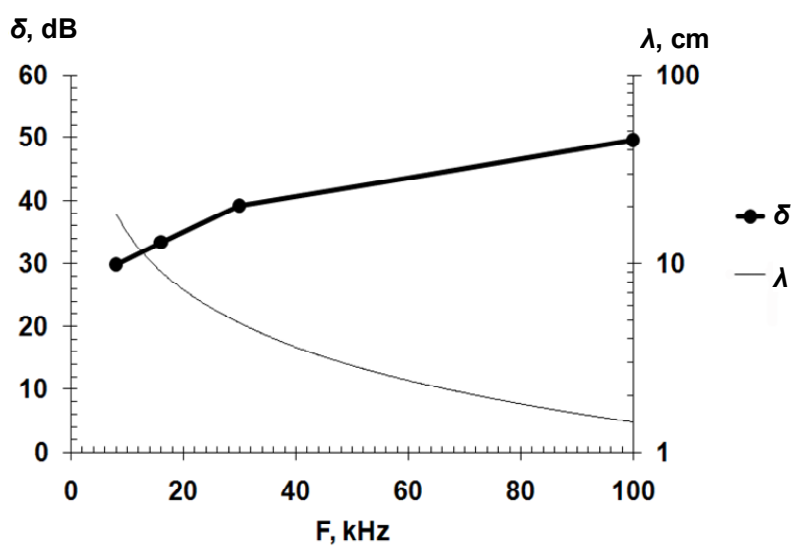

Figure 4. $(\delta)$ The ratios of mean values of the detection thresholds of stimuli at shielding of the mental foramens to the thresholds without shielding in dependence on the frequency of the stimuli spectrum maximum (F). The sound wavelength in water $(\lambda)$ in dependence on frequency $(F)$.

and right traveling wave antennas mutually intercross in ventral direction [21]. Due to this we can assume that in the medial plane of this area the beam patterns shape is mutually-complementary too.

The morphology features of the mental foramens that we considered in this study point out that their architecture is subordinated to the acoustical expediency. This natural inference in the main is confirmed by the experimental data reviewed below.

At shielding the mental foramens the mean values of detection thresholds of stimuli with spectrum maximum on frequencies $8,16,30$ and $100 \mathrm{kHz}$ increased on 30 , 34, 40 and $50 \mathrm{~dB}$, respectively (Figure 4). It means that the shielding significantly impairs the dolphin's hearing both on the low frequencies and on the frequencies of dolphin's whistling and in the echolocation frequencies range. This new original results testify that the mental foramens are the unique sound-conducting pathway into the fat body of the mandibular canal in the frequency range at least from $6 \mathrm{kHz}$ and up to $150 \mathrm{kHz}$ (in view of stimuli broadbandness). This fact does not give any chances for the sound conduction along the other hypothetical pathways $[1-3,9,11,12,17,18,20]$.

At the same time the detection thresholds decreases by $20 \mathrm{~dB}$ with increase in the wavelength of stimuli. This fact can be explained by increase in penetration of the sound beyond the bounds of the shield as a result of diffraction. Indeed, the sound wavelength significantly increases when the frequency lowers (Figure 4), and if on frequencies about $100 \mathrm{kHz}$ the shield length 10 times more of the wavelength, then for the frequencies about $16 \mathrm{kHz}$ the linear dimensions of the shield is becoming comparable to the wavelength and on frequencies of about $8 \mathrm{kHz}$ the dimensions of the shield are even shorter than the wavelength. Therefore, as it follows from the theory and the results (Figure 4, $100 \mathrm{kHz}$ ), in this waves lengths range, the shielding efficiency is increasing with increase of the ratio of the shield length to the wavelength.

Thus, if to take into account the diffraction effect, so becomes clear, that the shielding efficiency of the mental foramens would be identical if the ratio of the shield dimensions to the wavelength on the measured frequencies was constantly. It is also confirmed by the fact that the detection thresholds is vary in inverse proportion to the wave length, (Figure 4). Unfortunately, on the low frequencies it is difficult to perform the same ratio of the shield dimensions to the wavelength, as for frequency of $100 \mathrm{kHz}$, because the shield dimensions must be too big (for example, $1.875 \mathrm{~m}$, for the frequency of $8 \mathrm{kHz}$ ). Therefore, the efficiency of the used shield decreases with lowering frequency.

In view of obtained results the assumption that the morphological structures of lower jaw is the specialized peripheral part of dolphin's auditory system [21-25] that was based on the morphology study and modeling has now the additional experimental confirmation. Indeed, the affinity of the architecture of mental foramens and acoustics which the nature had created (Figure 3) apparently points out for us that the left and right row of mental foramens together with respective mandibular canal plays a role of external ear, if to use conventional terminology of a land mammal ear. But it already is qualitatively the new external ear implemented by the nature as the receive array and acoustical horn [21-25]. Moreover, each row of mental foramens being the receive array along with sound-conduction participates in the hearing directivity creation similarly to a land mammal pinna. Whereas, the mandibular canal being the acoustical horn plays a role of both matching device and part of the traveling wave antenna and like an external auditory meatus transmits a sound through a fat body to a tympanic bone's lateral wall [21-25] which plays a role of tympanic membrane [6,7,10,11,13-16].

The new external ear has apparently appeared in result of dolphins' ancestors' adaptation to new environment conditions, as evolutionary adaptation of the ear to the water and as functional adaptation of the ear in order that to fulfill the new more sophisticated functions in the structure of sonar.

Though the shielding efficiency of mental foramens on frequencies lower than $8 \mathrm{kHz}$ was not measured in this work, we can assume that the mental foramens are the unique way for the sound-conduction even on frequencies lower than $8 \mathrm{kHz}$. The constant inclination steepness of the low-frequency branch of a dolphin's audiogram (9-10 dB/octave) [29] from $50 \mathrm{~Hz}$ and up to 
$20-30 \mathrm{kHz}$ is evidencing in favor of this.

\section{CONCLUSIONS}

It is known that the Odontoceti experienced a number of functional and morphological modifications in the process of secondary adaptation to the aquatic habitat. Apparently, the new functions of mental foramens and mandibular canal in quality of the external ear that were discovered in this work belong to these modifications. The findings of this work give good reason to suppose the existence of the same external ear in Odontoceti.

\section{ACKNOWLEDGEMENTS}

Author would like to thank Sveta Yahno and Nadya Zhukova for the dolphin training. The experimental part of study was supported by the Ukrainian State fund of fundamental researches within the bounds of the joint project "sffr - rfbr - 2009 Ф28.4/024" with Russian Foundation for basic researches.

\section{REFERENCES}

[1] De Haan, F.V.R. (1957) Hearing in whales. Acta Otolaryngologica Supplementum, 134, 1-14.

[2] Fraser, F.C. and Purves, P.E. (1960) Hearing in the cetaceans: Evolution of the accessory air sacs and the structure and function of the outer and middle ear in recent cetaceans. Bulletin of the British Museum (Natural History), Zoology, 7(1), 1-140.

[3] Van Heel, W.H.D. (1962) Sound and cetacean. Netherlands Journal of Sea Research, 1(4), 407-507.

[4] Yamada, M. (1953) Contribution to the anatomy of the organ of hearing of whales. The Scientific Reports of the Whales Research Institute, 8(1), 1-79.

[5] Fleischer, G. (1973) Structural analysis of the tympanicum complex in the bottlenose dolphin (Tursiops truncatus). Journal of Auditory Research, 13, 178-190.

[6] McCormick, J.G., Wever, E.G., Palin, J. and Ridgway, S.H. (1970) Sound conduction in the dolphin ear. Journal of the Acoustical Society of America, 48(6), 1418-1428.

[7] Bullock, T.H., Grinell, A.D., Ikezono, E., Kameda, K., Katsuki, J., Nomota, M., Sato, O., Suga, N. and Yanagisawa, K. (1968) Electrophysiological studies of central auditory mechanisms in cetaceans. Zeitschrift für Vergleichende Physiologie, 59(2), 117-156.

[8] Airapetiantz, E.S., Voronov, V.A., Ivanenko, U.V., Ivanov, M.P., Ordovsky, D.L., Popov, V.V., Sergeev, B.F. and Chilingiris, V.I. (1973) The physiology of the sonar system in Black Sea dolphins. Zhurnal Evoliutsionnǒ Biokhimii $i$ Fiziologii, 9(4), 416-422.

[9] Dubrovsky, N.A. (1990) On the two auditory subsystems in dolphins: Sensory abilities of cetaceans. Plenum Press, New York, 233-254.

[10] Norris, K.S. (1964) Some problems of echolocation in cetaceans. In: Tavolga, W., Ed., Marine Bio-Acoustics, Pergamon Press, New York, 316-336.

[11] Norris, K.S. (1968) The evolution of acoustic mecha- nisms in odontocete cetaceans. In: Drake, E.T., Ed., Evolution and Environment, Yale University Press, New Haven, 297-324.

[12] Popov, V.V., Supin, A.Y., Klishin, V.O., Tarakanov, M.B. and Pletenko, M.G. (2008) Evidence for double acoustic windows in the dolphin, Tursiops truncates. Journal of the Acoustical Society of America, 123(1), 552-560.

[13] Ketten, D.R. (1997) Structure and function in whale ears. Bioacoustics, 8(1-2), 103-135.

[14] Nummela, S., Reuter, T., Hemila, S., Holmberg, P. and Paukku, P. (1999) The anatomy of the killer whale middle ear (Orcinus orca). Hearing Research, 133(1-2), 61-70.

[15] Hemilä, S., Nummela, S. and Reuter, T. (1999) A model of the odontocete middle ear. Hearing Research, 133(1-2), 82-97.

[16] Koopman, H.N., Budge, S.M., Ketten, D.R. and Iverson, S.J. (2006) Topographical distribution of lipids inside the mandibular fat bodies of Odontocetes: Remarkable complexity and consistency. Journal of Oceanic Engineering, 31(1), 95-106.

[17] Møhl, B., Au, W.W.L., Pawloski, J. and Nachtigall, P.E. (1999) Dolphin hearing: Relative sensitivity as a function of point of application of a contact sound source in the jaw and head region. Journal of the Acoustical Society of America, 105(6), 3421-3424.

[18] Brill, R.L., Moore, P.W.B., Helweg, D.A. and Dankiewicz, L.A. (2001) Investigating the dolphin's peripheral hearing system: Acoustic sensitivity about the head and lower jaw. Technical Report-1865, 1-14.

[19] Brill, R.L. (1988) The jaw-hearing dolphin: Preliminary behavioral and acoustics evidence. In: Nachtigal, P. and Moore, P., Ed., Animal Sonar. Processes and Performance, Plenum Press, New York, 281-287.

[20] Rimskaya-Korsakova, L.K. and Dubrovsky, N.A. (1998) Two sound conduction pathways to the cochlea participate information of spatial auditory image in the dolphin. Sensory Systems, 12(4), 497-506.

[21] Ryabov, V.A. (2004) Lower jaw-peripheric part of the dolphin echolocation hearing. In: Belkovich, V.M., Ed., Marine Mammals of the Holarctic, Collection of Scientific Papers, KMK Scientific Press, Moscow, 483-489.

[22] Ryabov, V.A. (2007) Mechanisms of a dolphin's echolocation hearing. In: Dible, S., Dobbins, P., Flint, J., Harland, E. and Lepper, P., Eds., Bio-Acoustics 2007, Proceedings of the Institute of Acoustics, 29(3), 283-293.

[23] Ryabov, V.A. (2008) Acoustic clutter field and echo reception by the dolphin. Biophysics, 53(3), 237-242.

[24] Ryabov, V.A. (2003) A dolphin lower jaw is a hydroacoustic antenna of the traveling wave. Journal of the Acoustical Society of America, 114(4), 2414-2415.

[25] Ryabov, V.A. (2008) Properties of the dolphin's mandible horn. In: Boltunov, A.N., Ed., Marine Mammals of the Holarctic, Collection of Scientific Papers, Astroprint Publishing House, Odessa, 468-473.

[26] Schusterman, R.J. (1980) Behavioral methodology in echolocation by marine mammals. In: Busnel, R.-G. and Fish, J.F., Ed., Animal Sonar Systems, Plenum Publishing Corporation, New York, 11-41.

[27] Agarkov, G.B., Homenko, B.G. and Hadjinsky, V.G. (1974) Morphology of dolphins. Naukova Dumka, Kiev, 1-167.

[28] Varanasi, U. and Malins, D.C. (1971) Unique lipids of the porpoise (Tursiops gilli): Differences in triacyclglyc- 
erols and wax esters of acoustic (mandibular and melon) and blubber tissues. Biochimica et Biophysica Acta, 231, 415-418.

[29] Johnson, C.S. (1967) Sound detection thresholds in marine mammals. In: Tavolga, W.N., Ed., Marine Bio-Acoustics II, Pergamon Press, Oxford, 247-260.
[30] Johnson, C.S. (1968) Maskes tonal thresholds in the bottlenose porpoise. Journal of the Acoustical Society of America, 44(4), 965-967.

[31] Babushina, E.S. (1999) Sound reception in marine mammals: Effects of stimulus parameters and transmission pathways. Biophysics, 44(6), 1064-1071. 